\title{
Commentary: Concomitant valvular intervention at the time (d) check for updates of ventricular assist device implant: Too much or not enough?
}

\author{
Vivek Rao, MD, PhD
}

\author{
From the Cardiovascular Surgery, Peter Munk Cardiac Centre, Toronto General Hospital, Toronto, Ontario, \\ Canada. \\ Disclosures: Author has nothing to disclose with regard to commercial support. \\ Received for publication Feb 25, 2019; accepted for publication Feb 26, 2019; available ahead of print April 6, \\ 2019. \\ Address for reprints: Vivek Rao, MD, PhD, Cardiovascular Surgery, Peter Munk Cardiac Centre, Toronto General \\ Hospital, 200 Elizabeth St, Toronto, Ontario, Canada M5G2C4 (E-mail: vivek.rao@uhn.ca). \\ J Thorac Cardiovasc Surg 2019;158:1090-1 \\ $0022-5223 / \$ 36.00$ \\ Copyright (c) 2019 by The American Association for Thoracic Surgery \\ https://doi.org/10.1016/j.jtcvs.2019.02.109
}

Durable mechanical circulatory support is now the standard of care for patients with advanced heart failure as either a bridge to transplant (BTT) or destination therapy (DT). The most recent Interagency Registry for Mechanically Assisted Circulatory Support report now suggests overall 2-year survival of $80 \%$ and $65 \%$ for BTT and DT, respectively. ${ }^{1}$ It is not surprising that survival in those patients who are not considered transplant candidates is poorer, because they are older and suffer from more comorbidities.

The decision to intervene on a cardiac valve at the time of left ventricular assist device (LVAD) implant remains controversial even from the era of pulsatile pumps. ${ }^{2}$ In this issue of the Journal, Suguira and colleagues, ${ }^{3}$ from the Texas Heart Institute, add further data to consider. Although the perioperative stroke rate was higher in the concomitant procedure group, overall survival was similar between those who had an isolated LVAD implant and those who had a concomitant procedure. Of note, in a subgroup survival analysis, it appears that mitral valve repair conferred a survival advantage. John and colleagues ${ }^{4}$ reported higher morbidity and mortality in patients receiving a HeartMate 2 device (Abbott Inc, Abbott Park, Ill) in addition to a concomitant valve intervention. In contrast, Maltais and colleagues ${ }^{5}$ examined both axial and centrifugal flow pumps (HeartWare, Medtronic Inc, Minneapolis, Minn) and found no survival differences between those who had an isolated ventricular assist device (VAD) implant versus a concomitant valve procedure.

The one valvular lesion that engenders widespread consensus is the management of aortic insufficiency. ${ }^{6}$ Most clinicians agree that the presence of moderate aortic insufficiency mandates intervention, although the type of intervention (repair vs replacement) continues to be controversial. It is clear that progressive aortic insufficiency in VAD recipients results in a "blind loop" whereby VAD output is circuitous from the outflow graft, retrograde via the incompetent valve, and back into the VAD inflow. As a result, true forward flow and systemic perfusion are

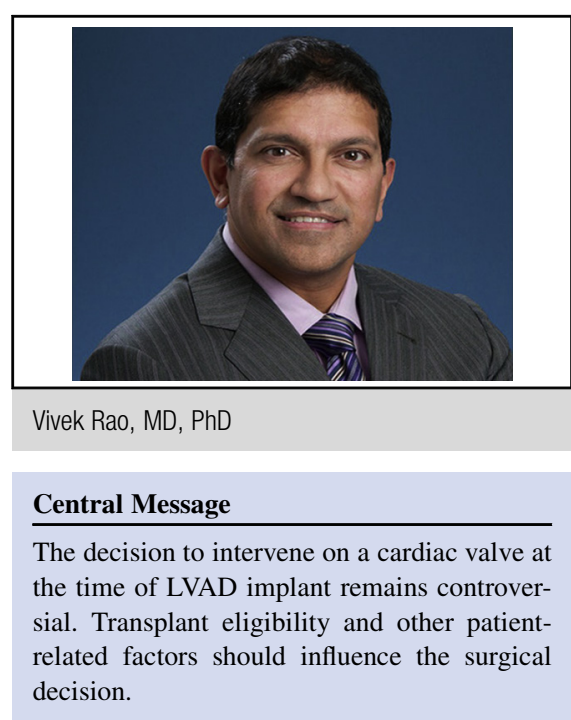

See Article page 1083 .

impaired. As aortic insufficiency develops/progresses with time, the presence of even mild aortic insufficiency should warrant consideration of repair in DT recipients, where transplant as a bail-out is not an option.

Mitral insufficiency usually improves after LVAD implant; however, several authors suggest that mitral repair can reduce pulmonary pressures and advocate concomitant intervention in patients who are bridged to transplant. ${ }^{3-5}$ However, the role of mitral valve repair in a DT population is less clear. Likewise, tricuspid valve repair has been advocated by those who think that right ventricular (RV) function and, by extension, renal function are improved in those LVAD recipients who present with severe tricuspid insufficiency. ${ }^{5}$ However, those patients with severe tricuspid insufficiency are likely to have worse preoperative RV function, and thus the results of concomitant tricuspid valve repair have not consistently shown benefit with respect to reducing RV failure or preserving renal function. Although the report by Suguira and colleagues $^{3}$ did not examine postoperative renal function stratified by type of valvular intervention, there was no difference in renal failure rates in the patients who had an isolated LVAD implant versus a concomitant procedure.

What is missing in all of the cited studies examining concomitant valvular surgery is the effect on transplant rates and post-transplant survival. Does a valvular 
intervention, particularly mitral or tricuspid repair, improve the rates of successful BTT and does concomitant mitral repair truly reduce post-transplant RV failure (presumably by an additive reduction in pulmonary vascular resistance over and above that of LVAD decompression)?

Furthermore, are the increased rates of morbidity and mortality a reflection of a DT population who are more likely to undergo aortic valve intervention than a BTT population? The present study by Suguira and colleagues ${ }^{3}$ does not stratify outcome by indication for VAD support, but it is worthy to note that more than $50 \%$ of their patients received implants with a BTT strategy.

The current report by Suguira and colleagues ${ }^{3}$ confirms that when indicated, valvular intervention can be performed with acceptable morbidity and mortality in LVAD recipients. As in several prior reports, we are not provided with information regarding outcomes in patients with valvular regurgitation who did not receive intervention, as well that effect on transplant outcomes. Sadly, we are still left with the controversial question when dealing with LVAD recipients who have significant valvular regurgitation: Is intervention too much or not enough?

\section{References}

1. Kormos RK, Cowger J, Pagani FD, Teuteberg JJ, Goldstein DJ, Jacobs JP, et al The Society of Thoracic Surgeons INTERMACS database annual report: evolving indications; outcomes and scientific partnerships. J Heart Lung Transplant. 2019; 38:114-26.

2. Rao V, Slater JP, Edwards NM, Naka Y, Oz MC. Surgical management of valvular disease in patients requiring left ventricular assist device support. Ann Thorac Surg. 2001;71:1448-53.

3. Sugiura T, Kurihara C, Kawabori M, Critsinelis AC, Wang S, Civitello AB, et al Concomitant valve procedures in patients undergoing continuous-flow left ventricular assist device implantation: a single-center experience. J Thorac Cardiovasc Surg. 2019;158:1083-9.

4. John R, Naka Y, Park SJ, Sai-Sudhakar C, Salerno C, Sundareswaran KS, et al, Impact of concurrent surgical valve procedures in patients receiving continuous flow devices. J Thorac Cardiovasc Surg. 2014;147:581-9.

5. Maltais S, Haglund NA, Davis ME, Aaronson KD, Pagani FD, Dunlay SM, et al Outcomes after concomitant procedures with left ventricular assist device implantation: implications by device type and indication. ASIAO J. 2016;62:403-9.

6. Cowger J, Rao V, Massey T, Sun B, May-Newman K, Jorde U, et al. Comprehensive review and suggested strategies for the detection and management of aortic insufficiency in patients with a continuous-flow left ventricular assist device. J Heart Lung Transplant. 2015;34:149-57. 